\title{
Distributed LQG control of a water delivery canal with feedforward from measured consumptions
}

\author{
João M. Lemos, Luís F. Pinto, Luís M. Rato and Manuel Rijo
}

\begin{abstract}
This work addresses the design of distributed LQG controllers for water delivery canals that include feedforward from local farmer water consumptions. The proposed architecture consists of a network of local control agents, each connected to one of the canal pools and sharing information with their neighbors in order to act in a coordinated way. In order to improve performance, the measurement of the outflow from each pool is used as a feedforward signal. Although the feedforward action is local, it propagates due to the coordination procedure. The paper presents the distributed LQG algorithm with feedforward and experimental results in a large scale pilot water delivery canal.

Index Terms-Distributed Control, distributed LQG control, feedforward, water delivery canals.
\end{abstract}

\section{INTRODUCTION}

\section{A. Motivation}

Water delivery canal systems are often spread over wide geographical areas, with actuators and local controllers in isolated spots [1], [2]. Furthermore, due to their physical characteristics, the use of pure decentralized control, $i . e$. structures in which local controllers act on the basis of pure local measurements, and without any exchange of information among them, may lead to poor performance or even instability. On the other way, completely centralized control architectures may not only be unfeasible due to the complexity of the transmission network involved, but highly unreliable as well, since communication links may be interrupted by hazardous causes.

These features provide a strong motivation for the employment of distributed control, in which a network of local control agents act in a coordinated way by communicating with their neighbors. If designed adequately, distributed control has a number of advantages:

- Simplicity of design of local controllers, conjugated with good overall performance;

- Increased reliability with respect to failures of either local control agents or communication links;

- Adequate management of local objectives, that may vary for canals crossing different administrative districts.

This work was supported by FCT (Portugal) under project AQUANET - Decentralized and reconfigurable control for water delivery multipurpose canal systems, contract PTDC/EEA-CRO/102102/2008, and INESC-ID multi-annual funding through the PIDDAC Program funds.

J. M. Lemos is with INESC-ID and IST/UTL, R. Alves Redol 9, 1000029 Lisboa, Portugal, jlmldinesc-id.pt

L. F. Pinto is with INESC-ID, Lisboa, Portugal.

L. M. Rato is with Dep. Eng. Inform., Univ. de Évora, Rua Romão Ramalho 59, 7000-671 Évora, Portugal, Imr@uevora.pt

M. Rijo is with NuHCC, Univ. of Évora, Pólo da Mitra apartado 94 7000-554 Évora, Portugal, rijo@uevora.pt
The objectives associated with the management and control of water delivery canal systems are manifold and depend on the type of canal and the way it is operated. Usually, saving water is a major aim, with automatic control known to increase canal efficiency [3]. In some cases, the water is driven to the canal only during the periods of usage, while in other situations of interest a minimum level of water must always be ensured, for instance for ecological reasons. In any case, in irrigation canals, the key point is to ensure that the desired amount of water, during a specified period of time, is available to the farmers that require it, while simultaneously water level constraints are respected, $e . g$. to avoid over floods that represent water spillage and may damage certain types of canals or to ensure a minimum water level. If translated in terms of control system specifications, this means that the water levels must follow given references, with specified transients, and rejecting disturbances due to water extraction by users.

In the situation considered in this paper, the main concern is to keep the water level downstream of each pool close to specified values, rejecting disturbances induced by offtake consumptions. Hence, local upstream control is used and complemented with feedforward from accessible disturbances. Although other schemes could be considered that lead to a more efficient use of water [3], the concern here is to demonstrate how a LQG controller including feedforward terms can be modified to act in a distributed setting to regulate water level in a multiple pool canal.

\section{B. Literature review}

Centralized LQG control of hydrosystems has long been considered [8], [9], [10], [11]. Model predictive control (MPC) provides an approximation to LQG that has the advantage of allowing the incorporation of constraints in a easy way, but requires a higher computational load. Applications of MPC to water delivery canals include both centralized [12] and distributed examples [13]. In [14] a decentralized version of LQG for a multicanal system has been obtained in a way that has a tight connection with predictive control. For that sake, the overall system is decomposed in a number of interacting subsystems. A local control agent is then associated with each subsystem, in which the manipulated variables are computed by minimizing a receding horizon quadratic cost using the LQG algorithm. The coordination between the controllers of the local subsystems is achieved using the decomposition-coordination approach, based on dual optimization [15]. Accordingly, the manipulated variables are computed, at the beginning of each sampling interval, by 